Este libro forma parte del acervo de la Biblioteca Jurídica Virtual del Instituto de Investigaciones Jurídicas de la UNAM www.juridicas.unam.mx 
Este libro forma parte del acervo de la Biblioteca Jurídica Virtual del Instituto de Investigaciones Jurídicas de la UNAM

\title{
DE POR QUÉ UN BUEN ANÁLISIS TEÓRICO PUEDE SER RELEVANTE. ¿CÓMO ENTENDER EL GONGEPTO DE VULNERABILIDAD EN TIEMPOS DE COVID?
}

Florencia LUNA

\begin{abstract}
SUMARIO: I. Introducción. II. ¿En qué consiste la visión tradicional? III. ¿Cómo se puede entender el concepto de vulnerabilidad? IV. ¿Cómo se relaciona esta concepción de la vulnerabilidad con la pandemia de COVID-19? V. Repensando la vulnerabilidad: los gatillos o condiciones estímulo. VI. Repensando la vulnerabilidad: el efecto cascada. VII. Repensando la vulnerabilidad: evaluación y práctica. VIII. Palabras finales.
\end{abstract}

\section{INTRODUCGIÓN}

Si bien el concepto de vulnerabilidad se discute y argumenta en la bioética - sobre todo al inicio - desde la ética de la investigación, también es un concepto fundamental para la ética de la salud pública. Desde ese lugar, tiene suma relevancia a la hora de pensar el impacto de la actual pandemia y sus conexiones con la vulnerabilidad.

En este trabajo presento un análisis teórico del concepto de vulnerabilidad. Además, argumento que es importante tener una correcta conceptualización del mismo y que el concepto "tradicional" de vulnerabilidad no es el más adecuado. Sostengo que mi propuesta no sólo resulta interesante teóricamente sino que también permite colaborar con la práctica para identificar, evaluar y priorizar ciertas vulnerabilidades. Además, trato de mostrar cómo mi concepción de vulnerabilidad sirve para dar cuenta de nuevas vulnerabilidades que se presentan con la actual pandemia.

En este artículo expongo, en primer término, lo que se ha considerado la concepción tradicional de este concepto. En segundo lugar, señalo algunos de los problemas que implica. Luego presento mi propia concepción de la noción de vulnerabilidad así como los últimos planteos teóricos que he 
Este libro forma parte del acervo de la Biblioteca Jurídica Virtual del Instituto de Investigaciones Jurídicas de la UNAM

formulado (el efecto cascada y las condiciones estímulo). También muestro cómo puede trabajarse con estos elementos teóricos en el ámbito práctico y cómo esta concepción de vulnerabilidad puede resultar interesante y dar cuenta de algunos desafíos que nos presenta la pandemia de COVID-19 actualmente.

\section{II. ¿EN QUÉ CONSISTE LA VISIÓN TRADICIONAL?}

La visión tradicional se basa en lo que se denomina el enfoque "subpoblacional". Con frecuencia se hace referencia a "poblaciones vulnerables". Esto se puede percibir por ejemplo en las Guías (CIOMS 2002). ${ }^{1} \mathrm{Y}$, si bien actualmente tenemos una nueva versión de estas pautas (CIOMS 2016), ${ }^{2}$ que toma en cuenta la propuesta que aquí analizaremos, ${ }^{3}$ esta concepción de subpoblación sigue estando presente en el lenguaje ordinario.

La concepción tradicional permea el lenguaje ordinario. Es más, el lenguaje cotidiano nos lleva a utilizar esta terminología ya que resulta más simple y supuestamente más "intuitivo". Sin embargo, aún si puede ser que resulte más fácil hablar en estos términos deberíamos tratar de ser más conscientes de sus implicancias.

¿Qué planteaban entonces las Pautas de la CIOMS? En el 2002 cuando prácticamente no se había cuestionado este concepto, estas guías consideraban la existencia de tres grupos de poblaciones vulnerables:

1. Aquellas/os con capacidad limitada para consentir, (niñas/os, personas con desórdenes mentales o prisioneros/as);

1 Consejo de Organizaciones Internacionales de las Ciencias Médicas (CIOMS) y Organización Mundial de la Salud (OMS), 2002, Pautas éticas internacionales para la investigación biomédica en seres humanos, Ginebra.

2 Consejo de Organizaciones Internacionales de las Ciencias Médicas (CIOMS) y Organización Mundial de la Salud (OMS) 2016 Pautas éticas internacionales para la investigación relacionada con la salud con seres humanos, Ginebra.

3 La última versión de las Pautas de la CIOMS toma mi concepción de vulnerabilidad si bien no utiliza el término "capas de vulnerabilidad". El artículo que presenta las nuevas Pautas (van Delden J. y van der Graaf R., "Revised CIOMS International Ethical Guidelines for Health Related Research Involving Humans", fAMA, 2016, 6 de diciembre, DOI:10.1001/jama.2016.18977) señala los cambios que se introducen en las Pautas del 2016, entre ellos señalan problemas en el concepto de vulnerabilidad y citan mi artículo (Luna, F. y Vanderpoel, S., "Not the usual suspects: addressing layers of vulnerability", Bioethics, vol. 27, núm. 6, 2013, pp. 325-332) como el enfoque adecuado que seguirán las Pautas. 
Este libro forma parte del acervo de la Biblioteca Jurídica Virtual del Instituto de Investigaciones Jurídicas de la UNAM

2. miembros subordinados (estudiantes de medicina, enfermería, soldados...), y

3. otros grupos o clases (personas en geriátricos, recibiendo ayuda social, desempleados/as, minorías étnicas, refugiados/as, pacientes con enfermedades incurables, etcétera).

Ahora bien, ¿qué problemas se plantearon a partir de esta visión tradicional basada en un enfoque poblacional? Esta concepción fue criticada por resultar demasiado abarcativa: prácticamente todas las personas pertenecían a grupos vulnerables. Esto generaba una superpoblación de grupos vulnerables.

Uno de los problemas señalados era que si se trataba de un concepto tan amplio y todos/as somos vulnerables, no tiene sentido establecer protecciones especiales (como se hace en investigación). Eso llevó a un grupo muy reconocido de bioeticistas estadounidenses ${ }^{4}$ a plantear que este uso tan amplio del concepto de vulnerabilidad era muy nebuloso como para tener sentido. Y, si bien, en parte sus críticas tienen sentido y se necesita una mejor conceptualización de este término; ellos terminan prácticamente dejando de lado este concepto que - a mi criterio- es fundamental.

Otro de los problemas de este enfoque sub-poblacional es que torna esencial al concepto de vulnerabilidad: brinda condiciones necesarias y suficientes para pertenecer o no a tales grupos. Esta concepción supone, además, una visión paradigmática del sujeto de investigación (aquella del adulto normal, relativamente alfabetizado, con una comprensión media, que puede consentir una investigación, etcétera). Este sujeto de investigación ideal o supuesto funciona como línea de base. Aquellos/as que no cumplen con este estereotipo se consideran como una población vulnerable. En este sentido una de las críticas que se formula es que rotula y estigmatiza ya que una vez que se establece que alguien es vulnerable es muy difícil salir de esa posición. A raíz de esto se puede caracterizar esta posición como funcionando con una metáfora de etiquetas. ${ }^{5}$ Por ello otra de las críticas a esta forma de conceptualizar la vulnerabilidad es que rotula y estigmatiza.

4 Levine, G., Faden, R., Grady, C., Hammerschmidt, D., Eckenwiler, L., Sugarman, J., The Limitations of "Vulnerability" as a Protection for Human Research Participants, American Fournal of Bioethics, 4 (3), 2004, pp. 44-49.

5 Luna, F., "Identifying and evaluating layers of vulnerability-a way forward", Developing World Bioethics, 19, 2019, p. 87. 
Este libro forma parte del acervo de la Biblioteca Jurídica Virtual del Instituto de Investigaciones Jurídicas de la UNAM

\section{III. ¿CÓMO SE PUEDE ENTENDER EL CONCEPTO \\ DE VULNERABILIDAD?}

En mi artículo "Elucidating the Concept of Vulnerability. Layers not labels" 6 critico esta visión tradicional y planteo, en cambio, que este concepto debe entenderse como dinámico y relacional. Allí argumento que no se trata de un concepto de "todo o nada", no debe ser considerado como una etiqueta, sino que está vinculado al contexto y a las circunstancias. Éstos delinean su alcance y las formas de concebirlo. Esta concepción alternativa a una visión basada en sub- poblaciones no brinda condiciones necesarias y suficientes, se trata de una visión no esencialista que desafía una visión rígida.

En ese artículo propongo pensar a este concepto a través de la metáfora de las capas de vulnerabilidad. Éstas son múltiples y diferentes, pueden quitarse capa por capa. No hay una "única y sólida vulnerabilidad" que agotaría la categoría. Por el contrario, pueden coexistir diferentes tipos de vulnerabilidades, diversas capas de vulnerabilidad volviendo a la persona más y más vulnerable.

\section{IV. ¿CÓMO SE RELACIONA ESTA CONCEPCIÓN DE LA VULNERABILIDAD CON LA PANDEMIA DE COVID-19?}

Mi concepción de la vulnerabilidad (basada en diferentes estratos y relacionada con el contexto) puede explicar por qué el personal de salud (médicos/ as, etcétera) puede considerarse vulnerable o susceptible de padecer una capa de vulnerabilidad con el COVID-19. Si bien, generalmente, se trata de un grupo de personas que tiene educación y ciertas posibilidades socio-económicas que los ubicaría, según la concepción tradicional, como un colectivo que no resulta vulnerable; esta pandemia los torna vulnerables. La situación contextual varía y la exposición a un virus tan contagioso así como las condiciones de los sistemas de salud, muchos de los cuales carecen de recursos adecuados de protección, hacen que el equipo de salud adquiera una capa de vulnerabilidad. Lo interesante es ver cómo con esta nueva conceptualización de la noción de vulnerabilidad se puede explicar claramente este fenómeno: la vulnerabilidad no es una propiedad esencial, el contexto la determina. Así

6 Luna, F., "Elucidating the Concept of Vulnerability. Layers not Labels", International Fournal of Feminist Approaches of Bioethics, vol. 2, núm. 1, 2009. Una versión en castellano es "Vulnerabilidad: la metáfora de las capas", Revista Lexis-Nexis, Furisprudencia Argentina, número especial, IV, 2008, Buenos Aires, pp. 60-67. 
Este libro forma parte del acervo de la Biblioteca Jurídica Virtual del Instituto de Investigaciones Jurídicas de la UNAM

dependiendo de las circunstancias ciertas poblaciones que habitualmente no consideramos vulnerables pueden llegar a serlo. ${ }^{7}$ Pero, como se mostrará más adelante, también permite explicitar que hay diferentes tipos o "grados" de vulnerabilidad.

Con el COVID-19 también vemos como si bien la población vulnerable consiste en - supuestamente los adultos mayores-, otros grupos como algunas mujeres, niñas, minorías étnicas o grupos de escasos recursos (a los que originalmente no se consideró población vulnerable porque son una población joven y, en teoría, pueden superar sin mayores inconvenientes el COVID-19) son tan o más afectados. Y esto se puede explicar fácilmente considerando que estas personas o grupos padecen una gran cantidad de capas de vulnerabilidad. La metáfora de las capas muestra que los grupos y personas que resultan vulnerables pueden ir variando. Según como resulte el contexto pueden acumular capas de vulnerabilidad y resultar cada vez más vulnerables. Por ejemplo, en EEUU las muertes por COVID-19 son altísimas en la comunidad afroamericana. En Milwaukee tres cuartos de las muertes se dieron en la población afroamericana y en Saint Louis (al momento de la escritura del editorial que se cita) todas las muertes salvo la de tres personas eran de afroamericanos. ${ }^{8}$ Así a la etnia, se le agrega la situación de pobreza, las dificultades para poder trabajar en modo de "teletrabajo", etcétera... Lo mismo sucede con muchas poblaciones originarias a las cuales se les añade que padecen de otras comorbilidades como la diabetes o problemas cardíacos. O la inmunodepresión de algunas personas con VIH/sida. De esta manera se puede comprender cómo la pandemia entrelaza un conjunto de situaciones que van volviendo más y más vulnerables a esas personas.

Otro ángulo que muestra la relevancia del contexto en el análisis de la vulnerabilidad es el impacto de las políticas de salud pública en las vidas de las personas y cómo estas políticas pueden tornar más vulnerables a ciertas personas. Por ejemplo, una política universalmente implementada fue el aislamiento social voluntario u obligatorio. El punto a considerar es la con-

7 Por ejemplo en el artículo Luna, F., "Vulnerabilidad y las mujeres de clase media: el caso de la sangre de cordón umbilical", Perspectivas Bioéticas, año 16, núm. 30, 2011. pp. 13-33, argumento algo parecido respecto de las mujeres embarazadas de clase media y el impacto que tiene en ellas la publicidad y los diferentes mecanismos de "imposición social" de los bancos de colecta de sangre de cordón umbilical.

8 Van Dorn, AV., Cooney, R. E., Sabin, M. L., "COVID-19 exacerbating inequalities in the US", The Lancet, 395 (10232), 2020, pp. 1243 y 1244, disponible en: https://wrere.ncbi.nlm. nih.gov/pmc/articles/PMC7162639/. 
Este libro forma parte del acervo de la Biblioteca Jurídica Virtual del Instituto de Investigaciones Jurídicas de la UNAM

secuencia que esta política puede implicar en ciertos grupos. Un dato relevante es el que el 23 de marzo del 2020 reportó UNICEF. ${ }^{9}$ Señaló que en Latinoamérica y el Caribe más de 154 millones de niños no estaban yendo, temporalmente, a la escuela. El punto va mucho más allá de la pérdida de la escolaridad, ya que 85 millones de niños de la región se benefician con planes alimenticios y para 10 millones de ellos resultan la fuente más confiable de alimentación diaria. Así no se trata sólo de comorbilidades, a esto hay que sumar otras dimensiones psico-sociales que explicitan esta dialéctica entre contexto, política pública y vulnerabilidad.

El abanico de posibilidades es bastante amplio. Por ejemplo, por el confinamiento se puede perder el trabajo y con ello dejar de tener recursos alimentarios (pensemos en los trabajadores informales). Téngase en cuenta que en la India más del $80 \%$ de los trabajadores son informales y cómo este tipo de restricciones impacta en la gente. Respecto de la informalidad, la dificultad de acatar el confinamiento y el peso de esto en las familias vemos que no se trata sólo de la India sino que también la misma situación afecta a la mayoría de nuestros países. La falta de recursos alimentarios puede traer otras consecuencias y retrotraernos al plano físico y médico, por ejemplo en Malawi existe un programa para dar tratamiento antirretroviral. Teniendo en cuenta el alcance a un millón de personas, en el 2018 se había llegado a cubrir un 79\% de las personas; ahora a raíz de la falta de seguridad alimentaria más de un $45 \%$ de las personas abandonaron el tratamiento antiretroviral. ${ }^{10}$

De esta manera se puede observar que la forma en la que la pandemia afecta a las diferentes personas y grupos va variando y, en algunos casos, se van adicionando más y más capas de vulnerabilidad. Así se puede percibir cómo se va sumando capa sobre capa, cada una implicando diferentes dimensiones (de salud, de género, social, económica... sobre aquellas personas que ya padecen co-morbilidades). En este sentido la metáfora de las capas permite dar cuenta de la multiplicidad de dimensiones involucradas y ya veremos cómo algunas de ellas pueden tener efectos especialmente nocivos. Estas situaciones nos llevarán - como se apreciará luego- a analizar cómo debemos evaluarlas y a cuáles dar prioridad. Puntos esenciales para la salud pública.

9 The Lancet, "Editorial. Redefining vulnerability in the era of Covid-19", The Lancet, (395), 4 de abril, 2020.

10 The Lancet HIV, "The syndemic threat of food insecurity and HIV", The Lancet HIV, $7(2), 2020, \mathrm{e} 75$. 
Este libro forma parte del acervo de la Biblioteca Jurídica Virtual del Instituto de Investigaciones Jurídicas de la UNAM

\section{REPENSANDO LA VULNERABILIDAD: LOS GATILLOS O CONDICIONES ESTÍMULO}

Recientemente, he vuelto a reflexionar sobre este concepto para avanzar en su elucidación y posible aplicación práctica. ${ }^{11}$ Así complementé lo que ya había trabajado desde el 2008 (aquí no presentaré la relación de mi concepción con diferentes definiciones sobre vulnerabilidad que se han brindado). ${ }^{12}$ En este trabajo, en cambio, me centraré en dos nuevos conceptos clave que introduzco en tal artículo. ${ }^{13}$ La idea era entender cómo funciona el concepto de vulnerabilidad desde la ética de la investigación y la evaluación de protocolos y también pensar cómo las políticas públicas afectan o inciden en las diferentes capas de vulnerabilidad que pueden superponerse y abarcar diferentes dimensiones. Por ejemplo, examinar si había algún criterio a tener en cuenta a la hora de evaluar y jerarquizar o decidir por donde comenzar: cuál capa de vulnerabilidad atacar primero. Uno de los objetivos era poder identificar las capas más dañinas y más probables para prevenir que operen. También creía relevante presentar algunas obligaciones ya sea para evitar exacerbar vulnerabilidades existentes como para intentar erradicar o minimizar tales capas.

En función de tales objetivos, en primer lugar argumenté que el concepto de vulnerabilidad es un concepto disposicional. Lo interesante de esta forma de abordaje es poder comprender cómo funciona el concepto. Ser vulnerable o padecer una capa de vulnerabilidad señala que una persona puede ser dañada, abusada, explotada, etcétera. Y habrá circunstancias que pueden hacer esto más o menos factible. Sin embargo, una persona no necesita ser dañada, abusada, explotada para ser considerada una persona vulnerable. Es más si se la daña, abusa o explota ya no es más vulnerable sino alguien dañado, abusado o explotado: ha sido vulnerado. Así pues, la posibilidad de ser vulnerado es lo relevante. Y esto es crucial. Lo que muestra es que la vulnerabilidad manifiesta una propiedad disposicional - que si bien fue reconocida por algunas autoras $-{ }^{14}$ no ha sido suficientemente destacado en el análisis y funcionamiento de este concepto.

\footnotetext{
11 Luna, F., "Identifying and evaluating layers of vulnerability-a way forward", Developing World Bioethics, 19, 2019, pp. 86-95.

12 Para una elaboración de ese punto véase Luna, F., "Identifying and evaluating layers of vulnerability-a way forward", Developing World Bioethics, 19, 2019, pp. 86-95.

13 Idem.

14 Hurst, S., "Vulnerability in research and health care. Describing the elephant in the room?”, Bioethics, 22 (4), 2008, pp. 191-202.
} 
Este libro forma parte del acervo de la Biblioteca Jurídica Virtual del Instituto de Investigaciones Jurídicas de la UNAM

Ahora bien, ¿qué características tienen los conceptos disposicionales? Un ejemplo clásico de disposicionalidad puede encontrarse en la propiedad de ser soluble. Un terrón de azúcar tiene esta propiedad. El mismo permanece sólido hasta que lo introducimos en líquido. Así esta propiedad permanece latente hasta que una condición estímulo (el hecho de colocar el terrón en líquido) gatilla la solubilidad.

Uno de los puntos que no se ha destacado suficientemente - y que yo considero fundamental - es la importancia de poder identificar cuál o cuáles son las condiciones-estímulo ("el gatillo o los gatillos") que desencadenarían una capa de vulnerabilidad... En el caso de la solubilidad del terrón de azúcar, la condición estímulo o el gatillo consistiría en la acción o el evento de sumergirlo en líquido.

Ahora bien, ¿ipor qué en nuestro análisis es esto relevante? Porque no necesariamente esas capas de vulnerabilidad se actualizarán, dependerá de que esos gatillos o condiciones estímulo estén presentes y funcionen. Y si podemos identificar cuáles son esos gatillos — si se pueden producir fácilmente o cuán probables son-, y podemos evitarlos o erradicarlos, podemos prevenir la vulneración de tales personas o grupos. De esta manera el análisis teórico nos alerta respecto de ciertos elementos que debemos considerar y buscar en la realidad para evitar que la vulnerabilidad se actualice y vulnere.

Así, si volvemos al caso del personal de salud y la capa de vulnerabilidad que adquieren en la actual pandemia, podemos señalar que la falta de equipos de protección personal adecuados funciona como una condición estímulo o un gatillo que fácilmente puede llevar a este colectivo a resultar dañado y por lo tanto vulnerado. Si identificamos cuál es la condición estímulo debemos evitarla, en este caso se debe insistir en la necesidad de brindar al equipo de salud los equipos de protección necesarios.

En esta situación en particular, la condición estímulo es claramente identificable y apunta a la necesidad de realizar acciones de protección que son relativamente simples o viables (si bien pueden requerir recursos económicos extra y una coordinación adecuada para obtenerlos); pero en otros casos estas condiciones estímulo pueden no ser tan claramente identificables o pueden resultar de muy dificil erradicación (pueden estar relacionadas a situaciones socioeconómicas que un investigador o aún ciertas políticas públicas no pueden modificar o erradicar). Sin embargo resulta fundamental examinar si existen tales condiciones estímulo, cuán probables son. Este concepto - a mi juicio crucial - no ha sido suficientemente subrayado en los análisis previos que se han formulado de la vulnerabilidad. 
Este libro forma parte del acervo de la Biblioteca Jurídica Virtual del Instituto de Investigaciones Jurídicas de la UNAM

\section{REPENSANDO LA VULNERABILIDAD: EL EFECTO CASCADA}

Un segundo concepto que considero es muy importante en la conceptualización de la vulnerabilidad es la identificación de aquellas capas que tienen lo que denomino "efecto cascada". Se trata de un concepto que retomo de unas autoras australianas feministas (Wendy Rogers, Susan Dodds y Catriona Mackenzie) que también analizan el concepto de vulnerabilidad en consonancia con mi propia propuesta. ${ }^{15}$

Rogers, Dodds y Mackenzie explicitan que hay una "fuente patogénica" de vulnerabilidad y dicen: "Algunas respuestas pueden exacerbar vulnerabilidades existentes o generar nuevas vulnerabilidades". También señalan que éstas pueden ser generadas por relaciones sociales y personales moralmente disfuncionales. ${ }^{16}$

Así la primera observación que se puede formular respecto del planteo anterior es que en la caracterización de estas vulnerabilidades se puede distinguir: a) sus efectos y b) su origen. En mi artículo propongo destacar a) sus efectos y denominarlas capas de vulnerabilidad con efecto cascada o vulnerabilidad cascada. Defiendo esta posición porque lo que a mi criterio resulta relevante es el efecto que una capa de vulnerabilidad pueda tener sobre otras ya existentes, o si tiene una suerte de efecto dominó (la situación de una capa de vulnerabilidad que genera otras capas) de manera tal de ir produciendo cada vez más vulnerabilidades. Y, si bien, en algunos casos estas capas o fuentes de vulnerabilidad pueden estar acompañadas de elementos disfuncionales esto, a mi juicio, no es lo más relevante. Un ejemplo de este tipo de vulnerabilidad puede señalarse en el caso de la falta de diagnóstico en enfermedades raras. Estas enfermedades son muy poco frecuentes y hay muy pocos casos en el mundo por lo tanto es habitual que no se diagnostiquen inmediatamente (y no puede atribuirse a mala intención o problemas en el sistema de salud). Sin embargo la falta de diagnóstico puede generar nuevas capas de vulnerabilidad: malos tratamientos o falta de tratamiento y el em-

15 Se trata de Wendy Rogers, Susan Dodds y Catriona Mackenzie. Ellas siguen mi propuesta de pensar el concepto de vulnerabilidad en términos de capas y tener en cuenta los aspectos contextuales (Véase: Rogers, W., Mackenzie, C., y Dodds, S., "Why bioethics needs a concept of vulnerability?", IfFAB, 5(2) 2012). Ellas proponen una taxonomía con la cual yo disiento (creo que vuelve rígido el planteo pero que además no resulta una buena taxonomía (Véase Meek Lange, M., Rogers, W., Dodds, S., "Vulnerability in Research Ethics. A way forward", Bioethics, vol. 27, number 6, 2013, pp. 330-340). En el artículo (Luna, F., "Rubens, corsets and taxonomies: a response to Meek Lange, Rogers and Dodds", Bioethics, vol. 26, number 6, 2015, pp. 448-450) planteo mis críticas. Sin embargo en ese último artículo también aparece el concepto de fuente patógena que considero debe ser repensado y retomado y que en mi propuesta se transformará en una capa de vulnerabilidad con efecto cascada.

16 Meek Lange, M., Rogers, W., Dodds, S., op. cit. 
Este libro forma parte del acervo de la Biblioteca Jurídica Virtual del Instituto de Investigaciones Jurídicas de la UNAM

peoramiento de la condición, la búsqueda de nuevos médicos o tratamientos y el agotamiento familiar, gastos excesivos para poder brindar un sostén al niño o niña con tal padecimiento, problemas familiares (debido a la sobredemanda ocasionada por la situación), nuevos embarazos y descendencia con una patología semejante (en muchos casos estas enfermedades tienen un origen genético), etcétera. Este caso muestra que lo fundamental es el impacto o el daño que este tipo de capa de vulnerabilidad genera. Lo fundamental es la multiplicación de vulnerabilidades. Además de que no necesariamente este efecto cascada surge por problemas sociales o personales patológicos.

Siguiendo esta línea de pensamiento podemos preguntarnos: ¿Qué capas de vulnerabilidad con efecto cascada podrían surgir por el COVID? Acá podemos analizar la política pública que involucra el confinamiento. Sobre todo en el caso del confinamiento obligatorio (en el cual se requiere permanecer en la casa las 24 horas durante meses (en Argentina, en la ciudad de Buenos Aires y gran Buenos Aires, al momento de redactar este trabajo se han transitado más de cuatro meses de confinamiento)). Lo primero que se puede señalar es que puede generar una serie de vulnerabilidades como lo es acrecentar la violencia doméstica, los abusos sexuales, el abuso de alcohol, el riesgo de embarazo no deseado y de embarazo adolescente (ya que no sólo puede afectar a la pareja adulta sino a las convivientes o hijas), etcétera. Por ejemplo, en la Argentina, las llamadas a la línea de teléfono por violencia doméstica se incrementaron en un $90 \%$ en ciudad de Buenos Aires desde que comenzó el aislamiento obligatorio. Y en la provincia de Buenos Aires se recibieron más de un $50 \%$ de llamadas por maltrato a niñas y niños según UNICEF Argentina. Lamentablemente, esto no es novedoso, un reporte de Naciones Unidas que analiza los efectos socioeconómicos del Ébola en África también subrayaba el incremento en el riesgo de embarazos en niñas, el abandono escolar y el abuso infantil. ${ }^{17}$ Así, una determinada política pública pensada para proteger a los ciudadanos puede generar cierto tipo de vulnerabilidades con efecto cascada y puede volver más y más vulnerables a ciertas personas de la comunidad por ejemplo a mujeres y a adolescentes...

\section{REPENSANDO LA VULNERABILIDAD: EVALUACIÓN Y PRÁCTICA}

En las dos secciones anteriores señalé que la vulnerabilidad es un concepto disposicional y que una característica de algunas capas de vulnerabilidad es

17 UN report on Ebola, disponible en: https://wrew.uneca.org/sites/default/files/PublicationFiles/eca_ebola_report_final_eng_0.pdf. 
Este libro forma parte del acervo de la Biblioteca Jurídica Virtual del Instituto de Investigaciones Jurídicas de la UNAM

la posibilidad de tener un efecto cascada. ¿Por qué son importantes estos conceptos? Al identificar la condición estímulo o aquello que gatillará una capa de vulnerabilidad o al determinar qué capas de vulnerabilidad pueden tener este efecto cascada podremos reconocer situaciones que pueden generar mayor daño y jerarquizarlas a la hora de pensar qué evitar o cómo minimizar o erradicar vulnerabilidades. En el caso anterior, podemos implementar paralelamente al confinamiento modelos de prevención de la violencia familiar, buscar lugares alternativos para algunas mujeres, establecer códigos de alerta y seguridad, etcétera.

Así, estos dos conceptos que a primera vista parecen meramente teóricos, tienen fuertes implicaciones prácticas. Al identificar qué capas tienen estos efectos multiplicadores o cuáles son los elementos que las pueden gatillar, podemos elaborar políticas públicas que los eviten o minimicen o podemos diseñar protecciones o empoderar a los sujetos de investigación. Esta propuesta permite establecer cierto orden y fijar prioridades: podemos jerarquizar aquellas capas de vulnerabilidad que se considera serán más dañinas y trabajar para evitarlas, minimizarlas o erradicarlas si fuera posible.

Pero nótese que no sólo estos últimos dos conceptos son sumamente relevantes, también la misma noción de capas de vulnerabilidad y la conceptualización que implica pensar que pueden haber capas diferentes superponiéndose nos lleva a tener una mirada más compleja. Y esto ocurre no sólo respecto de cómo se da la vulnerabilidad sino también respecto de poder pensar su multidimensionalidad, interseccionalidad y también poder elaborar múltiples y diferentes respuestas. Debajo de cada "etiqueta" que plantea el modelo tradicional hay múltiples capas y, quizá, cada una necesite de una respuesta diferente, algunas sean más nocivas que otras y algunas de ellas puedan ser gatilladas más inmediatamente. Considero, entonces, que esta conceptualización resulta fundamental para poder diseñar políticas públicas adecuadas que se puedan adaptar al contexto.

\section{PALABRAS FINALES}

Esta pandemia revolucionó nuestro modo de vivir, de interactuar y también de morir. ${ }^{18}$ En algunos países, todavía, estamos luchando para poder tener tal pandemia bajo control y restablecer algo parecido a la normalidad anterior...

18 Un tema que no se trató en este trabajo pero que también plantea serios problemas éticos es la muerte al quedar la persona aislada de sus afectos en un hospital, la dificultad de comunicarse con sus seres queridos, el duelo de la familia, etcétera. Y no sólo el impacto que tiene en la persona internada sino también en sus familiares, su posibilidad de duelo, etcéte- 
Este libro forma parte del acervo de la Biblioteca Jurídica Virtual del Instituto de Investigaciones Jurídicas de la UNAM

La respuesta desde la salud pública fue pensada para cierto tipo de sociedades y no se tuvo en cuenta - al menos inicialmente - los problemas que implica vivir en barrios hacinados, carentes de agua potable así como la falta de recursos sociales y/o económicos por los cuales no se puede no salir a trabajar. Esta primera respuesta parece suponer pensar a la vulnerabilidad con un enfoque poblacional esencialista. Se toma como sujeto vulnerable paradigmático a las personas mayores de 65 años cuyas biologías suelen no resistir los ataques de la COVID-19. Esta propuesta pierde de vista el funcionamiento del concepto de vulnerabilidad y su relación con diferentes contextos. Quizás en Europa o en sociedades envejecidas, en países en los cuales hay redes de contención para las personas con menos recursos (y la pobreza no es tan amplia ni tan profunda o corrosiva), los adultos mayores resultan los únicos vulnerables. Pero tal como se explicó, eso depende del contexto. En otras sociedades como las nuestras con grandes desigualdades, altos niveles de pobreza, pocos o insuficientes recursos de contención social, racismos y discriminaciones; las vulnerabilidades se manifiestan de otra manera. Al no percibir este dinamismo y la interacción con el contexto pareciera que tampoco se tuvo en cuenta el impacto que ciertas políticas públicas podían tener y la generación de mayores vulnerabilidades a raíz de ciertas condiciones estímulo y ciertas capas con efecto cascada.

Necesitamos repensar las vulnerabilidades que están implicadas no sólo desde el ámbito práctico sino también desde un plano teórico. Este análisis teórico de la vulnerabilidad debería permitir no sólo la conceptualización de este ambiguo y saturado término sino también debería ayudarnos a manejarnos en la práctica. Debe permitir identificar y evaluar correctamente las diferentes capas de vulnerabilidad para luego poder pensar cómo evitarlas o minimizarlas para lograr una respuesta lo más ética posible.

ra. En Argentina, en el CONICET se creó la Red de Cuidados, Derechos y Decisiones en el Final de la Vida (RIOSP). Esta elaboró un documento con recomendaciones específicas. 